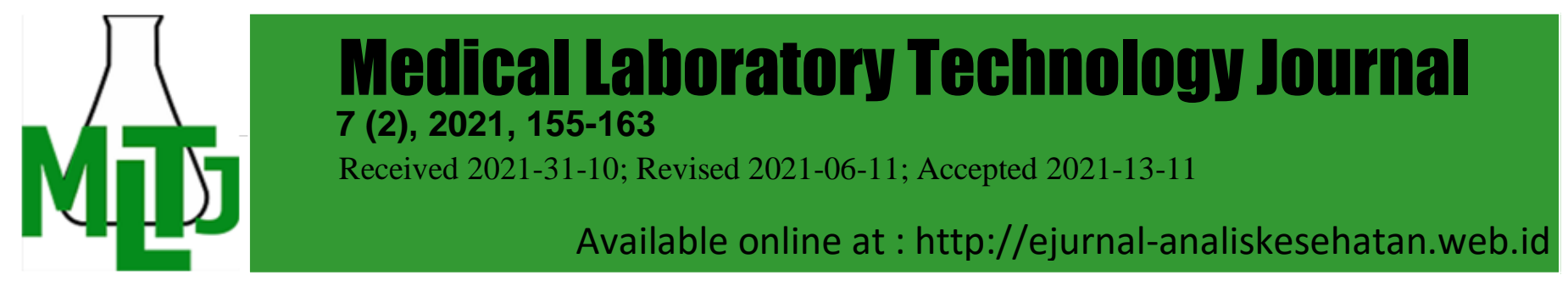

\title{
Effect of Diabetes Mellitus on the Excretory Function of the Liver
}

\author{
${ }^{*}$ Fidelis O. Oyakhire ${ }^{1}$ and Emokpae, M.A ${ }^{2}$, Enehizena O. Ogie ${ }^{2}$, Egho E. \\ Valentine $^{2}$
}

${ }^{1}$ Department of chemical pathology, Irrua Specialist Teaching Hospital, Irrua, Edo State, Nigeria. ' 2 Department of Medical Laboratory Science, University of Benin, Benin City, Edo State Nigeria. *Email: oyakhirefidelis@yahoo.com

DOI: $10.31964 / \mathrm{mltj} . \mathrm{v} 0 \mathrm{i} 0.416$

\begin{abstract}
Diabetes mellitus is an internationally recognized health problem and a leading cause of death worldwide. However, the most significant increase in prevalence is expected to occur in Asia and Africa, where most patients will be found by 2030. Diabetic Mellitus is a clinical and metabolic syndrome characterized by abnormal carbohydrate, protein, and fat metabolism resulting in hyperglycemia, increased protein breakdown, Ketosis or acidosis due to absolute or relative deficiency, and insulin resistance, thereby leading to vascular complications such as retinopathy, neuropathy, and nephropathy. This study evaluated the levels of plasma bilirubin, ALP, and GGT among diabetic patients. A total of eighty (80) individuals were recruited for the study comprising of forty (40) diabetes patients with age range 25-80 years and forty (40) control subjects with the age range of 20-30 years in Irrua/Ekpoma, Edo State, Nigeria. The study was carried out within six months (December 2018 - May 2019). All patients were diabetes. The serum Alkaline Phosphatase (ALP), gamma-glutamyl transferase (GGT), total and conjugated bilirubin were assayed by spectrophotometric method, and the data obtained were statistically analyzed using SPSS version 23.0 software. Serum levels of ALP, GGT, Total and unconjugated bilirubin were significantly elevated $(P<0.05)$ among diabetes patients than control subjects except for conjugated bilirubin which was lower $(P>0.05)$ when compared with that of the control subjects. The mean serum levels of ALP, GGT, total, unconjugated and conjugated bilirubin of male diabetes patients were non statistically significant $(P>0.05)$ when compared with female diabetes subjects except the age $(P<0.05)$. These findings indicate that hepatic injury was more likely among diabetes, and liver enzymes (ALP, GGT) are critical for monitoring glucose control concomitant with hepatic injury. Bilirubin is a potentially important biomarker for the assessment of the hepatic excretory system in diabetes mellitus.
\end{abstract}

Keywords: Diabetes mellitus; liver enzymes; hepatic injury.

\section{INTRODUCTION}

Diabetes mellitus is a metabolic disorder characterized by chronic hyperglycemia glycosuria, increased protein breakdown, ketosis, or acidosis, resulting from defective insulin action and secretion, leading to vascular complications such as retinopathy, neuropathy, and nephropathy (WHO, 1999). The role of the liver in DM has been long recognized because of its important place in carbohydrate metabolism. The loss of insulin effect on the liver leads to glycogenolysis and an increase in hepatic glucose production. World Health Organization projects that diabetic patients will exceed 350 million by 2030 (WHO, F1999). The prevalence value has not been uniform in Nigeria, though the International diabetic federation recorded 3.9\% in its current Atlas.

Corresponding Author: Fidelis O. Oyakhire

Department of chemical pathology, Irrua Specialist Teaching Hospital, Irrua, Edo State, Nigeria.

Email: oyakhirefidelis@yahoo.com 
Previous data have documented liver disease is a significant cause of morbidity and mortality of type 2 diabetes patients (Al-Jameil et al., 2014., Hanley et al., 2004). It is well known that the liver is a vital organ in the metabolism of carbohydrates and in maintaining glucose homeostasis during fasting and postprandial period ((AlJameil et al., 2014., Gavin, 1999).

Diabetic Mellitus (DM) leads to change in the liver-related to both function and morphology. ALP metabolizes phosphorus to bring energy to the body. In contrast, GGT brings energy to the tissues and enhances the metabolism of glutathione, the main thiol intracellular antioxidant agent in mammalian cells (Whitfield, 2001). Bilirubin is a yellow breakdown product of heme metabolism whose importance is antioxidant, removing peroxyl radicals generated by oxidative stress, which is associated with hyperglycemia state (Ndisang, 2009), thereby preventing diabetic vascular complications. Non-alcoholic fatty liver disease (NAFLD) is the scope of chronic liver disease in type 2 diabetes patients (Ballestri et al., 2016), which is characterized by excess deposition of fat in the liver and is associated with hepatic insulin resistance (Hanley et al.,2004) and type 2 Diabetes patients risk (Ballestri et al., 2016). Serum alkaline phosphatase (ALP),gamma-glutamyltransferase (GGT), and alanine aminotransferases (ALT) are suitable biomarkers of NAFLD. ALT and bilirubin have been considered the specific marker of liver injury, as found in high hepatocyte concentrations (Gianni, 2005), while GGT is present on the surface of most cell types and highly active in the liver, kidneys, and pancreas (Hanigan, 1996). Also, GGT is responsible for extracellular glutathione catabolism and may be linked to oxidative stress (Turgut, 2011) and chronic inflammation (Lee, 2004); both oxidative stress and chronic inflammation are essential pathways for hepatic insulin resistance (IR) and subsequently T2D development (Wang, 2016).

Previous data justified that diabetes causes hepatic injury such as hepatomegaly which in type I is related to an increase in the glycogen content. In contrast, type II is associated with fatty change, thereby leading to cytolysis and leakage out of the necrotic and damaged liver cells. Further data also reveals that the elevation of liver enzymes could express excess fat deposition in the liver, which is regarded as a feature of the insulin resistance syndrome (Chen,2008., Hammerman et al.,1998). There is clear evidence that cellular GGT level is closely related to oxidative stress indicators in vivo, either as an antioxidant or a pro-oxidant, depending on the circumstance (Schwertner,1994). It has been reported that many diabetes patients exhibit elevated levels of ALP, enzymes of the liver (ALT, AST, and GGT).

However, few studies reported that hepatic enzymes (ALP and GGT) are critical enzymes in monitoring diabetic Mellitus concomitant with liver injury and bilirubin are potentially essential biomarkers for assessing the hepatic excretory system in diabetes mellitus. Research is the first study carried out on diabetes patients in Esan people of Edo State, Nigeria. Hence, this case-control study was conducted to assess the effect of diabetes on the excretory function of the liver in a sample of Esan patients with diabetes mellitus.

\section{MATERIALS AND METHODS \\ Study Design and Population}

The study was carried out at Irrua, Esan Central Local Government and Ekpoma, Esan- West Local Government Area of Edo State, Nigeria. Irrua is a rural area located at latitude $6.7 \mathrm{~N}$ and longitude $6.2 \mathrm{E}$, with 105310 people. The people are farmers, traders, and Civil service workers. Ekpoma is a rural area located at latitude 6.5 Nand longitude $6.0 \mathrm{E}$, with 125842 people. The people are mainly farmers, traders, 
and civil service workers. A total of 80 subjects (male and female) comprised 40 diagnosed patients with diabetes mellitus and 40 healthy subjects as control. The patients were sampled at Irrua specialist teaching hospital, Irrua, while the healthy subjects mainly were students of AAU, Ekpoma. The study populations were divided into two groups, A and B.Group A included 40 subjects with diabetes mellitus, while Group B had 40 healthy subjects as control.

The research was designed to evaluate the serum levels of ALP, gammaglutamyl transferases (GGT) level, ALT, AST, and bilirubin (Total and indirect) in patients with diabetes mellitus with levels of the control subjects. The study was carried out within six (6) months (December 2018 - May 2019). Samples were collected twice-weekly (Mondays, Wednesdays). The patient's clinical diagnosis was confirmed by determining fasting blood sugar (FBS), which was above $126 \mathrm{mg} / \mathrm{dl}$. (7.0 $\mathrm{mmol} / \mathrm{l}$ ) while that of the apparently healthy subjects was confirmed by determination of fasting blood sugar (FBS), which was below $110 \mathrm{mg} / \mathrm{dl}(6.1 \mathrm{mmol} / \mathrm{l})$.

\section{Ethical consideration}

Ethical clearance was obtained from Edo State Ministry of Health, Benin City, Nigeria (ethical code 1208/157, dated 8th June 2018). Permission was obtained from the head of the department (Chemical pathology option), and informed consent was obtained from individual subjects before the commencement of the study.

Inclusion and exclusion criteria

Patients with diabetes mellitus were included while patients suffering from a hepatic disorder, alcoholic patients, pregnancy or use of oral contraceptives (in case of female subjects), and those using abused substances like hard drugs(barbiturate) that affects liver enzymes were excluded from the study.

Measurement of ALP, GGT, and Bilirubin

The Plasma Alkaline Phosphatase (ALP), gamma-glutamyl transferase (GGT) were measured enzymatically, total and conjugated bilirubin was measured by Jendrassik and Grof's method using V-730 UV-visible spectrophotometer (Easton, MD 21601, USA)

\section{Statistical Analysis}

Results obtained are presented as mean \pm standard deviation (SD). Data obtained were statistically analyzed using IBM SPSS Statistics version 23.0 software. The comparison of the mean values of measured parameters among diabetes subjects and control was performed using the Student t-test, the result obtained was compared at $95 \%$ confidence level $(p<0.05$-significance)

\section{RESULTS AND DISCUSSION}

The study was performed on eighty (80) subjects; subjects were divided into groups A and B. group A included forty (40) diabetes mellitus subjects, and Group B included forty (40) apparently healthy subjects. The results of the study are shown below. Table1 shows the comparison serum ALP, GGT, and bilirubin (total, conjugated and unconjugated) among diabetes and control subjects. It was observed that plasma ALP, GGT, total bilirubin, and unconjugated bilirubin were significantly elevated in diabetes mellitus than in control subjects $(P<0.05)$, While plasma conjugated bilirubin was lower in diabetes mellitus than control subjects $(P>0.05)$.

Table 2 compares the biochemical parameters (ALP, GGT, total bilirubin, conjugated, unconjugated bilirubin, and fasting blood sugar) among male and female diabetes and its control. It was observed that there were non statistically significant in the biochemical parameter $(P>0.05)$ 
Table 1 . Comparison of ALP, GGT, Total Bilirubin, Unconjugated and Conjugated Bilirubin between Diabetes and Control Subjects

\begin{tabular}{llll}
\hline Parameters & $\begin{array}{l}\text { Diabetes } \\
\text { subjects } \\
(\mathrm{n}=40)\end{array}$ & $\begin{array}{l}\text { Control } \\
\text { subjects } \\
(\mathrm{n}=40)\end{array}$ & $\mathrm{P}$-value \\
\hline ALP $(\mathrm{U} / \mathrm{L})$ & $183.83 \pm 11.68$ & $119.60 \pm 6.64$ & $\mathrm{P}<0.05^{\star *}$ \\
$\mathrm{GGT}(\mathrm{U} / \mathrm{L})$ & $26.85 \pm 10.96$ & $11.20 \pm 4.85$ & $\mathrm{P}<0.05^{\star *}$ \\
$\mathrm{~TB}(\mathrm{mg} / \mathrm{dl})$ & $1.29 \pm 0.58$ & $1.00 \pm 0.59$ & $\mathrm{P}<0.05^{\star *}$ \\
UCB $(\mathrm{mg} / \mathrm{dl})$ & $0.99 \pm 0.56$ & $0.63 \pm 0.51$ & $\mathrm{P}<0.05^{\star *}$ \\
CB(mg/dl) & $0.29 \pm 0.19$ & $0.37 \pm 0.28$ & $\mathrm{P}>0.05^{\star}$ \\
\hline
\end{tabular}

Values are expressed in mean $\pm S D$. ${ }^{* \star} \mathrm{P}<0.05$ Significant, ${ }^{\star} \mathrm{P}>0.05$ Non significant ALP-Alkalinephosphatase, GGT-Gamma-glutamyl transferase, TB-Total bilirubin, UCB-Unconjugated bilirubin, CBConjugated bilirubin.

Table 2. Comparison of ALP, GGT, Total Bilirubin, Unconjugated and Conjugated Bilirubin between Male and Female Subjects

\begin{tabular}{llll}
\hline Parameters & $\begin{array}{l}\text { Male diabetes } \\
\text { subjects } \\
(\mathrm{n}=11)\end{array}$ & $\begin{array}{l}\text { Female diabetes } \\
\text { subjects } \\
(\mathrm{n}=29)\end{array}$ & P-value \\
\hline ALP(U/L) & $170.64 \pm 3,73$ & $188.83 \pm 13.54$ & $\mathrm{P}>0.05^{*}$ \\
GGT $(\mathrm{U} / \mathrm{L})$ & $29.76 \pm 1.44$ & $25.35 \pm 9.36$ & $\mathrm{P}>0.05^{\star}$ \\
TB $(\mathrm{mg} / \mathrm{dl})$ & $1.33 \pm 0.68$ & $1.27 \pm 0.55$ & $\mathrm{P}>0.05^{\star}$ \\
UCB $(\mathrm{mg} / \mathrm{dl})$ & $0.99 \pm 0.65$ & $0.99 \pm 0.53$ & $\mathrm{P}>0.05^{\star}$ \\
CB $(\mathrm{mg} / \mathrm{dl})$ & $0.33 \pm 0.21$ & $0.27 \pm 0.18$ & $\mathrm{P}>0.05^{\star}$ \\
FBS $(\mathrm{mg} / \mathrm{dl})$ & $188.64 \pm 7.49$ & $192 \pm 7.9$ & $\mathrm{P}>0.05^{\star}$ \\
\hline
\end{tabular}

Values are expressed in mean $\pm S D .{ }^{* *} P<0.05$ Significant, ${ }^{*} \mathrm{P}>0.05$ Non significant ALP-Alkalinephosphatase, GGT-Gamma-glutamyl transferase, TB-Total bilirubin, UCB-Unconjugated bilirubin, CBConjugated bilirubin, FBS-Fasting blood sugar.

Diabetes mellitus (DM) is a fundamental health challenge and one of the primary causes of death worldwide; more than half of diabetes remains undiagnosed, primarily type 2 . The cause of this is insulin deficiency or relative insensitivity of insulin receptors. Without a timely diagnosis, the complication continues to be on the rise. According to $\mathrm{WHO}, 70 \%$ of diabetes reside in developing countries, to which Nigeria belongs. This research determined to evaluate the excretory function of the liver by assessing the liver enzymes(ALP, GGT) and bilirubin among diabetic subjects.

This study made statistical comparisons of plasma ALP, GGT, and bilirubin (Total, unconjugated and conjugated) for diabetes and apparently healthy control subjects. It was observed that the level of plasma ALP, GGT, total bilirubin, and unconjugated bilirubin were significantly increased with a $p$-value less than 0.05 $(P<0.05)$ while conjugated bilirubin was significantly decreased with a $p$-value greater than 0.05 (P>0.05) (Table1).

The significantly increased level of ALP as observed in diabetic Mellitus could be a result of the increased call for energy through ALP activity rather than the glycolytic and oxidative pathway of glucose six phosphates. Also, insulin deficiency or insulin resistance in DM reduced lipoprotein lipase activity and hepatic lipase responsible for the catabolism of lipoprotein (VLDL and chylomicron) and TG on HDL, thereby resulting from decreasing the clearance of remnant lipoprotein and 
accumulation of VLDL. The impaired ability of insulin to inhibit free fatty acid (FFA) release leads to enhance hepatic VLDL cholesterol production, which correlates with the degree of hepatic fat accumulation (Schielman,1975). The increased cholesterol content of the bile and increased gallbladder volume create a "primed environment for stone formation in the biliary tract that could result in partial or complete obstruction (Cholestasis) attributed to cytolysis and leakage out of the necrotic and damaged cell of the liver. It might also be attributed to the proliferation of the bile duct in the absence of obstruction, as seen in a diabetic cirrhotic patient.

This study was consistent with the result in (Falchuk 1993., Ginsberg, 1996. Sherlock,1985). They believed that diabetes causes hepatic injury such as hepatomegaly which in type I is related to an increase in the glycogen content. In contrast, type II is associated with fatty change, thereby leading to cytolysis and leakage out of the necrotic and damaged liver cells in diabetes mellitus.

In this study, plasma GGT level in diabetes patients was elevated compared with a control group, and the difference was highly significant $(P<0.05)$ statistically. The increase of GGT in diabetes mellitus could result from intrahepatic inflammation, an early marker of oxidation stress (Monami et al.,2008). One explanation for this observation is that the elevation of liver enzymes could express excess fat deposition in the liver, regarded as a feature of the insulin resistance syndrome (Marchesini et al.,2001., Malnick, 2003). There is clear evidence that cellular GGT level is closely related to oxidative stress indicators in vivo, either as an antioxidant or a pro-oxidant, depending on circumstance. (Hsueh,2003).

An increase in plasma GGT activity may respond to oxidative stress, making increased glutathione transport into cells, supporting a role of plasma GGT in the inflammation and oxidative stress. Another explanation might be related to liver response to pro-inflammatory cytokine TNF giving fatty hepatic change or the inflammatory processes accompanying non-alcoholic fatty liver and contributing to the systemic inflammation observed in these subjects frequently affected with metabolic syndrome (Perlstein et al., 2008). It might also be attributed to cholestasis (partial or complete blockage of the bile duct), resulting in cytolysis and leakage of necrotic and damaged liver cells. This study was consistent with the result in (Falchuk,1993., Ginsberg,1996., Lee et al.,2003., Monami et al.,2008., Sherlock,1985), they reported that the elevation of the hepatic enzyme (GGT) could be an expression of excess deposition of fat in the liver (intrahepatic inflammation) and oxidation stress associated with Diabetes Mellitus which therefore increase the metabolism of glutathione, a critical antioxidant defense thereby increasing the transport into the cell. They were also opinion that cytolysis and leakage out of necrotic and damaged liver cells in diabetes mellitus might be attributed to increasing plasma GGT.

In this study, Plasma total bilirubin in DM patients was elevated compared with a control group, and the difference was highly significant $(p<0.05)$ statistically. The increase of plasma total bilirubin in DM could result from an increased level of peroxyl radicals generated by oxidative stress, which is associated with the hyperglycaemic state. (Ndisang, 2009). Increase total bilirubin seen in this study is protective against coronary artery, stroke, and peripheral vascular diseases associated with metabolic syndromes and diabetes mellitus (Kern et al.,1973). Bilirubin has been regarded as a powerful endogenous antioxidant and anti-inflammatory agent (Chen, 2008., Stocker et al.,1987); thus, it stands to reason that increased total bilirubin level may be protective against autoimmune inflammation-related pathology of types I DM and oxidative physiologic stress associated with the development of type 2 DM. 
Current research also suggests that an increased level of total bilirubin block the product of various free radicals that might hinder the inhibitory response of the cell to take up the high glucose (Hammerman et al., 1998). This study was consistent with the result of a previous study (Lin et al., 2006., Perlstein et al.,2008., Schwertner,1994). They reported that an increased level of total plasma bilirubin is protective against peripheral vascular complications (Neuropathy, Nephropathy, and retinopathy) associated with oxidative stress in type II diabetes, thereby reducing the odds of incidence and prevalence of diabetes mellitus. This study shows that unconjugated bilirubin conveys the antioxidant effect, which protects against vascular complications. Also, there appears to be a beneficial effect of total bilirubin on diabetes.

In this study, plasma conjugated bilirubin in diabetes patients was lower when compared with a control group, and the difference was non statistically significant $(P>0.05)$. The decreased level of serum conjugated bilirubin in DM might be attributed to the problem of upstream excretion of bilirubin which reflects liver cell damage or bile duct damage within the liver itself, in which hemolysis, viral hepatitis, or cirrhosis can be suspected. Also, in the later stage of cholestasis, conjugated bilirubin may be elevated due to liver damage as a secondary effect of cholestasis.

Therefore, the hepatic fat accumulation may progress to fibrosis and cirrhosis, the most common clinical presentation is hepatomegaly, and diabetic patients have abnormal liver enzymes (ALP, GGT, transaminases) and normal conjugated bilirubin (Kern et al., 1973., Marchesini et al.,2001., Zimmerman et al., 1950). The mechanism of low/normal conjugated bilirubin is unclear but is perhaps related to diabetes cirrhotic condition.

From this study. It was also observed that the level of fasting blood sugar and ALP in diabetes females and males was not statistically significant, despite a slight rise in FBS and ALP in females than the male, which could be attributed to the effect of menopause and age. Previous studies reported by Ali (Ali, 2005., Ali, 2006) shows that elevated ALP in females diabetes was due to an increase of cholesterol content, high level of fat adiposity, and insulin deficiency (Ali, 2006., Martin, 2006) which is in agreement with this study. The presence of ALP in human pre-adipocytes is of interest that adipose tissue might be a source of serum ALP. Previous studies by Bin et al. (2021) reported a positive relationship between dyslipidemia and liver enzymes in diabetes; adipose tissue mass can influence the release of liver product into the circulation (increase ALP).

It was observed that the total serum bilirubin in male diabetes and female diabetes subjects were not statistically significant despite there being a rise in total bilirubin in male diabetes. A previous study done by Martin reported that it might be due to cardiovascular disease (coronary heart disease and myocardial infarction), which is commoner in males resulting from abnormal lipid (high plasma cholesterol and triglycerides and low high-density lipoprotein (HDL) among diabetes mellitus (Martin, 2006). Previous studies reported that the Increase concentration of serum bilirubin is in line with our finding In that it has an antioxidant effect on coronary artery disease (Breimer,1995., Hopkin et al.,1996., Schwertner,1994), stroke (Chen, 2008), and diabetic vascular complication (Inogochi et al.,2007).

The present study assesses the level of ALP as a biomarker of the excretion function of the liver. However, there are some limitations. First, our sample size may be small. Second, we measured the ALP and GGT once, which may not represent a long-term profile. Thus, further large sample sizes and repeat measurements of ALP 
and GGT are required to confirm if diabetes mellitus affects the excretion function of the liver.

\section{CONCLUSION}

Serum levels of ALP, GGT, total and unconjugated bilirubin were elevated, and low conjugated bilirubin was observed among diabetic subjects. This result shows that hepatic injury was more likely among diabetes patients. It is therefore suggested that hepatic enzymes (ALP and GGT) are critical enzymes in monitoring diabetes mellitus concomitant with liver injury and bilirubin are potentially essential biomarkers for the assessment of the hepatic excretory system in diabetes mellitus.

\section{ACKNOWLEDGEMENT}

The authors are particularly grateful to God, the giver of knowledge, and the Irrua Specialist hospital staff for their cooperation during the study.

\section{CONFLICT OF INTEREST}

The authors have not declared any conflict of interest.

\section{REFERENCES}

Ali, A.T., Penny, C.B. \& Paiker, J.E. (2005). Alkaline phosphatase is involved in the control of adipogenesis in the murine preadipocyte cell line, 3T3-L1. Clinical chemistry. Acta, 354, 101-109.

Ali, A.T., Penny, C.B. \& Paiker, J.E. (2006). The effect of alkaline phosphatase inhibitor on intracellular lipid accumulation in preadipocyte isolated from human mammary tissues. Annal Clinical Biochemistry, 43, 207-213

Al-Jameil, N., Khan, F. A., Arjumand, S., Khan, M.F. and Tabassum, H. (2014). Associated Liver Enzymes with Hyperlipidemic Profile in Type 2 Diabetes Patients. International Journal of Clinical and Experimental Pathology, 7, 43454349

Ballestri, S., Zona, S., Targher, G., Romagnoli, D., Baldelli, E., Nascimbeni, F., et al.: (2016). Nonalcoholic Fatty Liver Disease Is Associated with an Almost Twofold Increased Risk of Incident Type 2 Diabetes and Metabolic Syndrome. Evidence from a Systematic Review and Meta-Analysis. Journal of Gastroenterology and Hepatology, 31, 936-944. https://doi.org/10.1111/jgh.13264

Bin Dahman, L., Humam, M., Barahim, O. , Barahman, O. \& Balfas, M. (2021). Association between Liver Enzymes and Dyslipidemia in Yemeni Patients with Type Two Diabetes Mellitus. Journal of Diabetes Mellitus, 11, 41-51 doi: $10.4236 / j \mathrm{dm} .2021 .112004$.

Breimer, L.H. (1995). Serum bilirubin and risk of ischaemic heart disease in middle age British men. Clinical Chemistry, 41, 1504-1545.

Chen, Y.H., Chau, L.Y. \& Chen, J.W. (2008). Serum bilirubin and ferritin level link haeme oxygenase 1 gene promoter polymorphism and susceptibility to coronary artery disease in diabetes patient. Diabetes care, 31(8),1615-1620

Falchuk. K.R. \& Colin S. (1993). The intestinal and Liver complication of diabetes mellitus. Advance Internal Medicine, 38, 269-286

Gavin, N. and Levinthal, A.S.T. (1999). Liver Disease and Diabetes Mellitus. Clinical Diabetes, 17(2)

Giannini, E.G., Testa, R. and Savarino, V. (2005). Liver Enzyme Alteration: A Guide for Clinicians. Canadian Medical Association Journal, 172, 367-379. https://doi.org/10.1503/cmaj.1040752 
Ginsberg, H.N.(1996). Diabetic dyslipidemia: basic mechanism underlying the common hypertriglyceridemia and low HDL cholesterol levels. Diabetes, 645(suppl 3), S27 -S30

Hammerman, C., Goldstein, R., Kaplan, M., Eran, M. \& Goldschmide, D. (1998) . Bilirubin in the premature: toxic waste or natural defence? Clinical Chemistry, 44(12), 255-2553

Hanigan, M.H. \& Frierson Jr., H.F.(1996), Immunohistochemical Detection of GammaGlutamyl Transpeptidase in Normal Human Tissue. Journal of Histochemistry \& Cytochemistry,44, 1101-11108. https://doi.org/10.1177/44.10.8813074

Hanley, A.J., Williams K., Festa, A., Wagenknecht, L.E., D'Agostino, Jr., R.B., Kempf, J., et al. (2004), Elevations in Marker of Liver Injury and Risk of Type 2 Diabetes-The Insulin Resistance. Atherosclerosis Study. Diabetes, 53, 26232632, https://doi.org/10.2337/diabetes.53.10.2623

Hopkins, P.N., Wu, L.L., Hunt, S.G. \& Jame B.G. (1996). Higher serum bilirubin is associated with decreased risk for early familiar coronary artery disease. Arteriosclerosis Thrombosis Vascular Biology, 16, 250-255.

Hsueh, W.A. \& Quinones, M.J. (2003). Role of endothelial dysfunction in insulin resistance. American Journal Cardiology, 92,10-17

Inogochi, T., Sasaki, S., Kobayashi, K. \& Takayanagi, R. (2007). Relationship between Gilbert syndrome and prevalence of vascular complications in patients with diabetes. JAMA, 298, 1398-1400

Kern, W.H., Herger, A. H., Payne, J. H. \& Dewind, L.T. (1973). Fatty metamorphosis of liver in morbid obesity. Archives of Pathology and Laboratory Medicine, 96, 342-346

Lee, D. H., Ha, M. H., Kim, J. H. \& Christiani, D. C. (2003). Gamma-glutamyl Transferases and diabetes: A 4 years follow up study. Diabetologia, 46, 359364

Lee, D.H. \& Jacobs Jr., D.R. (2004). Association between Serum Gamma-Glutamyl transferase and C-Reactive Protein. Atherosclerosis, 178, 327-330. https://doi.org/10.1016/i.atherosclerosis.2004.08.027

Lin, J.P., Donell, C.J., schwager, J.P. \& Cupples, L.A. (2006). Association between the UGT1A1 28 allele, bilirubin levels, and coronary heart disease in the Framingham Heart study. Circulation, 114(14), 1476-1481.

Malnick, S.D., Bzergabe., M. \& Knobler, H. (2003). Non - alcoholic fatly liver: a common manifestation of a metabolic disorder. Quarterly Journal of Medicine, 96, 699-709

Marchesini, G., Brizi, M., Blanchi, G. \& Tomassetti, S. (2001). Nonalcoholic fatty liver disease a feature of the metabolic syndrome. Diabetes, 50, 1844-1850

Martin, A.C. (2006). Cardrovascular risk factors. In a textbook of clinical chemistry and metabolic medicine $\left(7^{\text {th }}\right.$ ed) publisher. Hodder Arnold. pp. 322

Monami,M., Barchini,G.,Lamma,K.\& Pala, L. (2008). Liver enzymes and risks of diabetes and cardiovascular disease. Metabolism, 57(3), 387-392

Ndisang, J.F., Lane, N. and Jadhav A. (2009). Upregulation of the haeme oxygenase system ameliorates postprandial and fasting hyperglycaemia in type 2 diabetes. Ameican Journal of Physiology- Endocrinology and Metabolism, 296(3), E1029-104.

Perlstein, T.S., Pande, R.L., Beckman, J.A. \& Creager, M.A. (2008). Serum total bilirubin level and prevalent lower - extremity peripheral arterial disease: National Health and Nutrition Examination Survey (NHANES) 1999 - 2004. Arteriosclerosis Thrombosis vascular Biology, 28(1), 166 
Schielman, J.D., Goodman, S.I., Mace, J.W.\& Patrick, A. (1975). Glutathionuria inborn error of metabolism due to tissue deficiency of gamma - glutamyl transpeptidase. Biochemical and Biophysical Research Communication, 65(1), 68-74. Doi:10.1016/S0006 - 291 (75) 80062

Schwertner, H.A., Jackson, W.G. \& Tolan, G. (1994). Association of low serum concentration of bilirubin with increased risk of coronary artery disease. Clinical Chemistry, 40, 18-23.

Sherlock,S. (1985). Diseases of liver and biliary tract. Published by Blackwell Scientific Publication. Pp. 386-387

Stocker, R., Yamamoto, Y., McDonagh, A.F. \& Glazer, A.N. (1987). Bilirubin is an antioxidant of possible physiological importance. Science, 235,1043-6

Turgut, O. \& Tandogan, I. (2011). Gamma-Glutamyltransferase to determine Cardiovascular Risk: Shifting the Paradigm Forward. Journal of Atherosclerosis and Thrombosis, 18, 177-181

Wang, Y., Koh, W., Yuan, J. \& Pan, A. (2016). Association between Liver Enzymes and Incident Type 2 Diabetes in Singapore Chinese Men and Women. British Medical Journal Open Diabetes Research and Care, 4, e000296 https://doi.org/10.1136/bmjdrc-2016-000296

Whitfield, J.B. (2001). Gamma-glutamyl transferase. Critical Review Clinical laboratory science, 38, 263-355

World Health Organization. (1999). Definition, Diagnosis and Classification of Diabetes Mellitus and Its Complications: Report of a WHO Consultation. Part 1, Diagnosis and Classification of Diabetes Mellitus. World Health Organization, Geneva, https://apps.who.int/iris/handle/10665/66040

Zimmerman, H. J., MacMurray, F. G., Rapaport, H. \& Alpert, L. K. (1950). Studies of liver in diabetes mellitus. structural and functional abnormalities. Journal of Laboratory and clinical medicine, 36, 912-921 